BMJ Open Ophthalmology

\title{
Optic perineuritis
}

\section{Sukriti Gupta, ${ }^{1}$ Prabhpreet Sethi, ${ }^{2}$ RamKrishan Duvesh, ${ }^{1}$ Harinder Singh Sethi, ${ }^{1}$ Mayuresh Naik (D) , ${ }^{3}$ Harminder $\mathrm{K} \mathrm{Rai}^{4}$}

\section{ABSTRACT}

Optic perineuritis (OPN) is a rare inflammatory disorder in which the inflammation is confined to optic nerve sheath. It can be idiopathic or secondary to underlying systemic autoimmune disorder. It usually presents with unilateral progressive diminution of vision with pain on eye movements and optic disc oedema. Hence, clinically OPN mimics optic neuritis resulting in delayed diagnosis and suboptimal treatment. In contrast to optic neuritis, patients with OPN are usually of older age group and more likely show sparing of central vision. MRI is an important tool for diagnosis of OPN apart from optic nerve sheath biopsy. Perineural enhancement on MRI is diagnostic of OPN. Oral corticosteroid therapy gives dramatic and rapid improvement in signs and symptoms. Rapid tapering of steroids increases the risk of relapse. Overall, prognosis of OPN is generally good if adequate treatment is given timely.

\section{INTRODUCTION}

Optic perineuritis (OPN), also known as perioptic neuritis, is a rare form of orbital inflammatory disease in which the optic nerve sheath is inflamed, resulting in marked thickening due to non-specific fibrosis ${ }^{1}$ as opposed to the inflammation of the optic nerve axons in optic neuritis $(\mathrm{ON})$. It is usually isolated and idiopathic in nature (primary OPN) but can occur secondary to infections and autoimmune diseases (secondary OPN) such as Antineutrophil Cytoplasmic antibodies (ANCA)-associated vasculitis, ${ }^{2}$ sarcoidosis, ${ }^{34}$ syphilis ${ }^{56}$ and Crohn's disease. ${ }^{7}$ First described by Edmunds and Lawford, ${ }^{8}$ OPN usually presents with pain, disc oedema and various features of optic nerve dysfunction. Radiologically, OPN may simulate optic nerve sheath meningioma, ${ }^{9}$ but clinically, it more often mimics acute demyelinating $\mathrm{ON}$, thus resulting in late diagnosis and treatment. It is important to distinguish between these two entities due to differences in their treatment and prognosis.

In the past, OPN was originally described as an optic neuropathy with optic disc oedema but without optic nerve dysfunction in the setting of normal intracranial pressure. ${ }^{10}$ This often occurs with syphilis infection of the optic nerve. ${ }^{11}$ However, with the emergence of modern neuroimaging techniques (MRI) that enabled imaging of the nerve sheath, the clinical and radiological features of OPN were revised.

\section{METHOD OF LITERATURE SEARCH}

We conducted a systematic review using the Cochrane methodology and reported the findings according to Preferred Reporting Items for Systematic Reviews and MetaAnalyses. CINAHL, EMBASE, Cochrane Library, MEDLINE, OTSeeker, PEDro and PsycINFO databases were searched using the terms 'optic neuritis', 'optic perineuritis', 'demyelinating' and 'multiple sclerosis'. Related definitions and descriptions were extracted. Two independent reviewers determined whether the studies met inclusion the inclusion criteria: reviews or randomised controlled trials; categorised interventions based on the name, core components and diagnostic populations; rated the quality of evidence and determined the strengths of recommendations using the GRADE criteria; and made recommendations using the evidence alert traffic light system.

\section{PATHOGENESIS}

Edmunds and Lawford described two forms of OPN: an exudative form, in which there is localised non-suppurative pachymeningitis; 912 and a purulent form, in which the leptomeningitis extends to involve the subarachnoid space around the optic nerve. In such cases, optic nerve function is considered to be normal as the inflammatory infiltrate is loosely organised around the optic nerve.

The common pathological reaction in OPN involves marked inflammatory thickening of the optic nerve sheath due to non-specific fibrosis. ${ }^{9}$ Visual loss in OPN has been attributed to secondary ischaemic infarction of the optic nerve due to circumferential compression of the optic nerve periphery by the mass of the thickened optic nerve sheath. ${ }^{13}$ Various stages of predominantly lymphocytic infiltration of the sheath and adjacent optic nerve parenchyma are usually present in biopsy specimens. Few authors have reported the presence of foci having degenerating 
(necrobiotic) collagen and vasculitic changes in the optic nerve sheath in cases of chronic inflammation. ${ }^{13}$ In their study, Dutton and Anderson showed the presence of granulomatous inflammation in the optic nerve sheath of one out of four patients. ${ }^{9}$ In chronic cases, the perivascular lymphocytic infiltration of the small optic nerve vessels (vascultits) has also been identified in a few patients. ${ }^{14}{ }^{15}$ Some cases have immune-mediated inflammation within the nerve sheath along with a few axons of optic nerve being affected. The degree and location of the optic nerve sheath inflammation defines the range of signs and symptoms seen in the disease. However, most cases are idiopathic.

\section{RISK FACTORS}

OPN is usually seen in older patients as compared with $\mathrm{ON}$, which is more prevalent in the middle age group. Purvin et al reported a case series in 2001 involving 14 patients with OPN, where the mean age of presentation was 41 years. ${ }^{1}$ OPN is more common in women. Unlike $\mathrm{ON}$, OPN is not associated with multiple sclerosis.

\section{AETIOLOGY}

Most cases of OPN are idiopathic, isolated and include primary OPN. However, many cases have been reported to occur secondary to systemic disorders such as Myelin Oligodendrocyte protein (MOG) antibody-associated demyelinating disease mimicking typical multiple sclerosis, sarcoidosis, ${ }^{34}$ giant cell arteritis (GCA),${ }^{16}$ tuberculosis, ${ }^{17}$ herpes simplex virus, ${ }^{18}$ herpes zoster virus, ${ }^{17}$ leukaemia, ${ }^{19}$ syphilis, $^{5}{ }^{6}$ wegener's granulomatosis ${ }^{2}$ primary or metastatic malignancy ${ }^{20}$ Crohn's disease, ${ }^{7}$ IgG4-related disease ${ }^{20}$ and neuroretinitis. ${ }^{21}$ Lai et al stated that majority of patients with OPN were diagnosed with Behcet's disease, suggesting that OPN might be a precursor to other autoimmune diseases. ${ }^{21} 22$

\section{CLINICAL FEATURES}

Patients with OPN often present with acute vision loss progressing over several weeks and eye pain exacerbated with eye movement similar to $\mathrm{ON}$, but the pain is more severe or longer lasting. Visual loss can range from none to severe, described by patients as blurring of vision, dimming, splotches or 'spots' in vision. ${ }^{1}$ On fundus examination, OPN patients have disc oedema in the affected eye. Usually, there is sparing of central vision with defects in the visual field such as paracentral scotomas, arcuate defects, centrocaecal scotomas, peripheral islands and altitudinal defects, and rarely, central scotomas. In addition, patients have signs and symptoms of optic nerve dysfunction, such as the possible presence of an relative afferent pupillary defect dyschromatopsia and poor contrast sensitivity. Unlike isolated ON, OPN may present with orbital signs and symptoms such as ophthalmoplegia, ptosis and exophthalmos, ${ }^{17}$ and rarely with intraocular lesions such as retinal necrosis, scleritis and episcleritis.

OPN is often clinically difficult to differentiate from $\mathrm{ON}$ and other inflammatory conditions because of their similar signs and symptoms. However, it is important to differentiate between the two entities due to their different therapeutic and prognostic implications. Patients with ON are at increased risk of developing multiple sclerosis and need to be counselled accordingly, whereas OPN is not associated with a risk for developing multiple sclerosis but is associated with recurrent visual

\begin{tabular}{|c|c|c|}
\hline Features & Optic perineuritis & Optic neuritis \\
\hline Age & Older & Younger \\
\hline Onset of visual loss & Subacute (over weeks) & Acute (days) \\
\hline Pathology & Optic nerve sheath inflammation & Optic nerve inflammation \\
\hline Visual field defect & Usually paracentral scotoma/ arcuate defect & Central scotoma \\
\hline Association with multiple sclerosis & No & Yes \\
\hline Signs & Less dyschromatopsia. Subtler RAPD & $\begin{array}{l}\text { More } \\
\text { Usually substantial RAPD }\end{array}$ \\
\hline Diagnosis: MRI findings & $\begin{array}{l}\text { Perineural enhancement ('tram-track' } \\
\text { sign on axial view and 'doughnut sign' on } \\
\text { coronal view) } \\
\text { Fat streakiness may also be present }\end{array}$ & Intraneural enhancement \\
\hline Treatment & Oral corticosteroids & $\begin{array}{l}\text { Intravenous methylprednisolone followed by } \\
\text { oral steroids }\end{array}$ \\
\hline Response to corticosteroids & Vision improves dramatically & $\begin{array}{l}\text { Steroids may speed the recovery but do not } \\
\text { affect the final visual outcome }\end{array}$ \\
\hline Relapse with steroids therapy & $\begin{array}{l}\text { Risk of relapse increases if the duration of } \\
\text { treatment is inadequate }\end{array}$ & $\begin{array}{l}\text { High risk of relapse with oral steroids if used } \\
\text { alone }\end{array}$ \\
\hline Prognosis & $\begin{array}{l}\text { Progressive deterioration of vision without } \\
\text { treatment with steroids }\end{array}$ & $\begin{array}{l}\text { Recovers spontaneously, even without steroid } \\
\text { therapy }\end{array}$ \\
\hline
\end{tabular}

RAPD, relative afferent pupillary defect. 
loss. In addition, the treatment recommended for OPN (oral glucocorticoids) is contraindicated in ON because of the chance of recurrence of $\mathrm{ON}$ with oral steroids. The key differences between ON and OPN are shown in table 1 .

\section{DIAGNOSIS}

OPN is diagnosed on the basis of clinical and radiographic findings. ${ }^{1}$ Ideally, to detect the classic perineural enhancement optic nerve sheath in OPN, a fat-suppressed, Gadolinium-enhanced T1 weighted MRI of the orbits should be performed. ${ }^{9}$ Unlike ON, wherein optic nerve enhancement is seen on MRI, the characteristic finding in OPN is the contrast enhancement of the optic nerve sheath with sparing of the optic nerve. This perioptic enhancement in OPN appears as 'Tram Tracks' in the axial view and as a 'Doughnut' in the coronal view. ${ }^{1}$ However, this tram track sign is not specific to OPN as it can also be seen in other inflammatory or neoplastic diseases affecting the optic nerve sheath, such as optic nerve sheath meningioma, sarcoidosis, lymphoma, leukaemia, orbital pseudotumor, perioptic haemorrhage and metastasis. ${ }^{23}$ Optic nerve sheath meningioma can be differentiated on the basis of calcifications, which can be seen using CT if the diagnosis is unclear. ${ }^{3}$ It is important to not confuse the tram track sign with normal enhancement of the dural sheath due to its rich vascular supply. ${ }^{23}$ Other findings that can be seen in OPN are the subtle enhancement of the sclera and extraocular muscles, which may present as a mild motility limitation leading to diplopia and the streaky enhancement of orbital fat. ${ }^{1}$ A CT scan can also be done to examine these features, but its spatial resolution cannot sufficiently differentiate intraneural enhancement from perineural enhancement. ${ }^{1}$ During the pre-MRI era, OPN was misdiagnosed as 'atypical corticosteroid-responsive ON'. (table 2)

The diagnosis of OPN can be confirmed with optic nerve biopsy, but it is usually not routinely indicated. ${ }^{17}$ It is usually useful in patients showing no response to corticosteroids and in those with no defining underlying aetiology for OPN. ${ }^{17}$ Optic nerve sheath inflammation

Table 2 Key differences between typical and atypical optic neuritis (ON)

\begin{tabular}{|c|c|}
\hline Typical ON & Atypical ON \\
\hline $\begin{array}{l}\text { Acute to subacute onset promoting over a several hours to } 2 \\
\text { weeks }\end{array}$ & $\begin{array}{l}\text { Severe visual loss (no light perception) which progress for }>2 \\
\text { weeks from onset }\end{array}$ \\
\hline $\begin{array}{l}\text { Young adult patient with peak manifestation between } 15 \text { and } \\
50 \text { years of age }\end{array}$ & Age $>50$ or $<12$ years \\
\hline \multicolumn{2}{|l|}{ Females $>$ males } \\
\hline Periocular pain (90\%) especially with eye movement & - Painless/painful/persistent pain $>2$ weeks \\
\hline $\begin{array}{l}\text { Unilateral loss of visual acuity variable in severity (from } 20 / 20 \\
\text { in } 10.5 \% \text { to no light perception in } 3.1 \% \text { ) or may be bilateral } \\
\text { usually in children often associated with a post or para } \\
\text { infectious demyelination }\end{array}$ & Simultaneous or sequential bilateral ON \\
\hline $\begin{array}{l}\text { Normal (65\%) or swollen (35\%) (more common in children) } \\
\text { optic nerve head } \\
\text { Possibility of mild uveitis and retinal periphlebitis } \\
\text { Reduced contrast sensitivity } \\
\text { Uhthoff's phenomenon (exercise or heat-induced deterioration } \\
\text { of visual symptoms) } \\
\text { Pulfrich phenomenon (misperception of the direction of } \\
\text { movement of an object) }\end{array}$ & $\begin{array}{l}\text { Abnormal ocular findings including: } \\
\text { Noticeable anterior and/or posterior segment inflammation } \\
\text { Significant uveitis and retinal periphlebitis } \\
\text { Intensely swollen optic nerve head } \\
\text { Severe optic disc haemorrhages } \\
\text { Retinal exudates } \\
\text { Macular star } \\
\text { Absence of any visual recovery within } 3-5 \text { weeks or continued } \\
\text { exacerbation in visual function }\end{array}$ \\
\hline Previous history of ON or MS & $\begin{array}{l}\text { Lower risk of developing MS } \\
\text { Family history }\end{array}$ \\
\hline $\begin{array}{l}\text { Spontaneous visual improvement in }>90 \% \\
\text { No deterioration in vision after steroids discontinuation }\end{array}$ & $\begin{array}{l}\text { Deterioration in vision after steroids discontinuation } \\
\text { Poor or no response to treatment with systemic steroids } \\
\text { Exquisitely steroid sensitive or steroid dependent optic } \\
\text { neuropathy }\end{array}$ \\
\hline $\begin{array}{l}\text { Ipsilateral RAPD. Lack of the defect suggests a preexisting or } \\
\text { concurrent optic neuropathy in the fellow eye } \\
\text { Visual field defect any type; ranging from commonly seen } \\
\text { diffuse depression and central or centrocecal scotoma to } \\
\text { rarely seen quadrantic and altitudinal defects }\end{array}$ & Manifestation of systemic diseases other than MS \\
\hline
\end{tabular}

Pallor of the optic disc

Optic atrophy lacking history of ON or MS

MS, Multiple sclerosis; RAPD, relative afferent pupillary defect. 
is evidenced by predominantly lymphocytic infiltration and/or detection of perineural fibrous tissue. ${ }^{19}$

Apart from neuroimaging, other laboratory tests can be performed in cases where no clear underlying aetiology can be detected. These include serological tests for syphilis ${ }^{24}$; serum ACE for sarcoidosis ${ }^{25}$; Mantoux test and chest X-ray for tuberculosis ${ }^{15}$; ANCA, IgG4, erythrocyte sedimentation rate for GCA, ${ }^{26}$ granulomatosis with polyangitis ${ }^{27}$ and Behcet's disease ${ }^{22}$ and lumbar puncture CSF analysis to rule out central nervous system malignancy and infection. Thus, evaluation of patients with suspected infectious optic neuropathy may include testing complete blood count; erythrocyte sedimentation rate; $\mathrm{C}$ reactive protein; serological testing; blood cultures; PCR or antibody assessment of aqueous humour, vitreous, serum or cerebrospinal fluid; a tuberculin skin test and/or quantification; tomodensitometry and MRI. Since serum ACE has low yield for the detection of neurosarcoidosis, patient evaluation should include a chest CT or Positron emission tomography (PET)-CT scan of the body. Similarly, the evaluation for tuberculosis would include an Interferon-Gamma Release Assay (IGRA) test in addition to chest imaging.

\section{DIFFERENTIAL DIAGNOSIS}

1. Demyelinating ON.

2. Orbital inflammatory syndromes (idiopathic, sarcoidosis, granulomatous).

3. Neoplasms (optic nerve sheath meningioma, leukaemia, lymphoma).

4. Infectious causes (tuberculosis, syphilis).

5. Posterior scleritis, secondary disc oedema associated with uveitis.

6. MOG antibody-associated demyelinating disease mimicking typical multiple sclerosis.

\section{MANAGEMENT}

The main treatment for OPN is corticosteroid therapy, which causes rapid and dramatic improvement in signs and symptoms typically within hours or within a day. However, recurrence has been reported with a short course of treatment, thus requiring a longer course of treatment with very slow tapering of the dose. The dosage depends on the frequency and rate of recurrence. ${ }^{28}$ Few cases have been reported that showed no improvement in vision even with corticosteroid therapy. ${ }^{22}$ This was attributed to chronic inflammatory infiltration along with fibrous tissue deposition in the dural sheath causing compressive optic neuropathy and ischaemic infarction. ${ }^{31}$ In a case study conducted by Purvin $e t a l$, indomethacin was used instead of steroids and was able to successfully treat two patients. ${ }^{1}$ Although steroids are the mainstay of OPN therapy, radiation therapy and immunosuppressants such as azathioprine have also been reported to be successful for refractory cases. ${ }^{17}$

OPN is not self-limiting; that is, it does not resolve without treatment. Corticosteroid therapy improves final visual outcomes in OPN unlike in $\mathrm{ON}$, while treatment with intravenous steroids only speeds up the visual recovery but does not affect final visual outcomes. ${ }^{1}$ Additionally, the dose and route of steroid administration differ. The preferred mode of treatment for OPN is oral and requires a higher dose of steroids $(80 \mathrm{mg}$ / day), whereas in $\mathrm{ON}$, the preferred mode is intravenous since there is a higher risk of recurrence reported with the use of oral steroids in ON. A short course of treatment used in ON (approximately 2 weeks) is generally not long enough to achieve lasting remission in OPN. ${ }^{1}$ The relapse of symptoms after cessation of therapy is more common in OPN. Hence, if a patient treated for ON shows relapse after therapy, the possibility of OPN should be considered.

\section{PROGNOSIS}

OPN usually has a relatively good visual prognosis. Unlike $\mathrm{ON}, \mathrm{OPN}$ is not associated with multiple sclerosis. The prognosis is influenced by the gap between the onset of visual loss and the initiation of steroid therapy. Another factor that influences prognosis is the frequency of recurrent attacks. OPN secondary to Behcet's disease has been reported to have a poor prognosis. ${ }^{22}$ A long duration of treatment with high-dose steroids can lead to complications in OPN.

\section{CONCLUSION}

OPN is a rare heterogeneous disease presenting with inflammation restricted to the optic nerve sheath rather than being more extensive within the orbit, as in other orbital inflammatory diseases. ${ }^{3}$ An older patient presenting with the classic triad of pain, optic neuropathy (relative afferent pupillary defect, loss of colour vision and contrast sensitivity), and optic disc swelling should be examined for possible OPN, especially if central vision is spared. A full history, as well as an ocular and systemic examination should be performed to rule out any underlying systemic disease. A fat-suppressed, gadolinium-enhanced T1-weighted MRI of the brain and orbit is of utmost importance for the diagnosis of OPN. It is useful for differentiating OPN from ON, other orbital inflammatory diseases and neoplasms. Optic nerve sheath biopsy is indicated in refractory cases and if the underlying aetiology is not known. Once OPN is diagnosed, high-dose oral steroids are administered for a period of months. Poor prognosis has been reported in cases with delayed initiation of treatment. Hence, early diagnosis and treatment of OPN are important.

Contributors All the authors were involved in the concept and design of the study, data acquisition, data analysis and interpretation, drafting manuscript, technical support and final review of the manuscript.

Funding The authors have not declared a specific grant for this research from any funding agency in the public, commercial or not-for-profit sectors.

Competing interests None declared.

Patient consent for publication Not required.

Provenance and peer review Not commissioned; externally peer reviewed. 
Open access This is an open access article distributed in accordance with the Creative Commons Attribution Non Commercial (CC BY-NC 4.0) license, which permits others to distribute, remix, adapt, build upon this work non-commercially, and license their derivative works on different terms, provided the original work is properly cited, appropriate credit is given, any changes made indicated, and the use is non-commercial. See: http://creativecommons.org/licenses/by-nc/4.0/.

ORCID iD

Mayuresh Naik http://orcid.org/0000-0003-0368-042X

\section{REFERENCES}

1 Purvin V, Kawasaki A, Jacobson DM. Optic perineuritis: clinical and radiographic features. Arch Ophthalmol 2001;119:1299-306.

2 Purvin V, Kawasaki A. Optic perineuritis secondary to Wegener's granulomatosis. Clin Exp Ophthalmol 2009;37:712-7.

3 Meehan K, Rodman J. Ocular perineuritis secondary to neurosyphilis. Optom Vis Sci 2010;87:E790-6.

4 Delaney P. Neurologic manifestations in sarcoidosis: review of the literature, with a report of 23 cases. Ann Intern Med 1977;87:336-45.

5 Toshniwal P. Optic perineuritis with secondary syphilis. J Clin Neuroophthalmol 1987;7:6-10.

6 O'Connell K, Marnane M, McGuigan C. Bilateral ocular perineuritis as the presenting feature of acute syphilis infection. $J$ Neurol 2012;259:191-2.

7 McClelland C, Zaveri M, Walsh R, et al. Optic perineuritis as the presenting feature of Crohn disease. J Neuroophthalmol 2012;32:345-7.

8 Edmunds W, Lawford JB. Examination of optic nerve from cases of amblyopia in diabetes. Trans Ophthalmol Soc U K 1883:3160-2.

9 Dutton JJ, Anderson RL. Idiopathic inflammatory perioptic neuritis simulating optic nerve sheath meningioma. Am J Ophthalmol 1985;100:424-30.

10 Miller NR, Newman NJW. Walsh and Hoyt's clinical neuroophthalmology. 5 edn. Baltimore, MD: Williams \& Wilkins, 1998.

11 Miller N, Newman N, Biousse V. Hoyt's clinical neuro-ophthalmology. 6 edn. Philadelphia, PA: Lippincott Williams \& Wilkins, 2005: 337-8.

12 Margo CE, Levy MH, Beck RW. Bilateral idiopathic inflammation of the optic nerve sheaths. Ophthalmology 1989;96:200-6.
13 Rush JA, Kennerdell JS, Donin JF. Acute periscleritis - a variant of idiopathic orbital inflammation. Orbit 1982;1:221-30.

14 Zhang TL, Shao SF, Zhang T, et al. Idiopathic inflammation of optic nerve simulating optic nerve sheath meningioma: CT demonstration. $J$ Comput Assist Tomogr 1987;11:360.

15 Morotti A, Liberini P, Padovani A. Bilateral optic perineuritis as the presenting feature of giant cell arteritis. BMJ Case Rep 2013;2013 doi:10.1136/bcr-2012-007959. [Epub ahead of print: 29 Jan 2013].

16 Bergman O, Andersson T, Zetterberg M. Optic perineuritis: a retrospective case series. Int Med Case Rep J 2017;10:181-8.

17 Byon IS, Jung JH, Kim HY, et al. Optic perineuritis secondary to acute retinal necrosis. J Neuroophthalmo/ 2013;33:419-21.

18 Townsend JH, Dubovy SR, Pasol J, et al. Transient optic perineuritis as the initial presentation of central nervous system involvement by pre-B cell lymphocytic leukemia. J Neuroophthalmol 2013;33:162-4.

19 Hickman SJ. Optic perineuritis. Curr Neurol Neurosci Rep 2016;16:16:16.

20 Wals KT, Ansari H, Kiss S, et al. Simultaneous occurrence of neuroretinitis and optic perineuritis in a single eye. $J$ Neuroophthalmol 2003;23:24-7.

21 Lai C, Sun Y, Wang J, et al. Optic perineuritis in Behçet disease. $J$ Neuroophthalmol 2015;35:342-7.

22 Kanamalla US. The optic nerve tram-track sign. Radiology 2003;227:718-9.

23 Pakdaman MN, Sepahdari AR, Elkhamary SM. Orbital inflammatory disease: pictorial review and differential diagnosis. World J Radiol 2014;6:106-15

24 Yu-Wai-Man P, Crompton DE, Graham JY, et al. Optic perineuritis as a rare initial presentation of sarcoidosis. Clin Exp Ophthalmol 2007;35:682-4.

25 Jacob M, Kodjikian L, Ponceau B, et al. [Can optic perineuritis be triggered by Mycobacterium tuberculosis?]. J Fr Ophtalmol 2006;29:e7.

26 Takazawa T, Ikeda K, Nagaoka T, et al. Wegener granulomatosisassociated optic perineuritis. Orbit 2014;33:13-16.

27 Tatsugawa M, Noma H, Mimura T, et al. High-dose steroid therapy for idiopathic optic perineuritis: a case series. J Med Case Rep 2010;4:404.

28 Hykin PG, Spalton DJ. Bilateral perineuritis of the optic nerves. $J$ Neurol Neurosurg Psychiatry 1991;54:375-6. 